\title{
Control of single-photon transport in a one-dimensional waveguide by another single photon
}

\author{
Wei-Bin $\operatorname{Yan}^{1,2}$ and Heng Fan ${ }^{2}$, 田 \\ ${ }^{1}$ Liaoning Key Lab of Optoelectronic Films \& Materials, \\ School of Physics and Materials Engineering, Dalian Nationalities University, Dalian, 116600, China \\ ${ }^{2}$ Beijing National Laboratory for Condensed Matter Physics, \\ Institute of Physics, Chinese Academy of Sciences, Beijing 100190, China
}

\begin{abstract}
We study the controllable single-photon transport in a one-dimensional (1D) waveguide with nonlinear dispersion relation coupled to a three-level emitter in cascade configuration. An extra cavity field was introduced to drive one of the level transitions of the emitter. In the resonance case, when the extra cavity does not contain photons, the input single photon will be reflected, and when the cavity contains one photon, the full transmission of the input single photon can be obtained. In the off-resonance case, the single-photon transport can also be controlled by the parameters of the cavity. Therefore, we have shown that the single-photon transport can be controlled by an extra cavity field.
\end{abstract}

PACS numbers: 03.67.Hk, 03.65.-w

\section{INTRODUCTION}

Single photons are considered as one of the most suitable carriers for quantum information. It is important to control the single-photon transport in quantum information processing. Recently, controllable single-photon transport in 1D waveguide with linear and nonlinear dispersion relations has been extensively investigated both theoretically [1 28] and experimentally 29 37]. In a 1D waveguide, the photons are confined to propagating only forward or backward in 1D space. By coupling an emitter to the waveguide, the strong photon-emitter interaction can be obtained and the photon transport in the $1 \mathrm{D}$ space can be affected by the interaction. It is known that for a 1D waveguide coupled to a two-level emitter [1, 2], the injected single photon will be completely reflected in the resonance case due to the interference between the wave function of the input photon and the spontaneously emitted photon. The single-photon transport in a 1D waveguide coupled to a multi-level emitter [17, 22] has also been studied. Compared to a two-level system, the multi-level system provides more controllable parameters. For instance, when a $1 \mathrm{D}$ waveguide is coupled to a $\Lambda$-type emitter, a strong pulse was employed to drive one of the atomic transitions. Since the single photon transport can be controlled by the extra pulse, the all-optical device can be achieved. However, most of the controls need strong pulse containing many photons or rely on other parameters. It is interesting to study the controllable single-photon transport by another photon without the classical field.

In this paper, we propose a scheme to study the control of single-photon transport in a 1D waveguide with nonlinear dispersion relation at the single-photon level.

*Electronic address: hfan@iphy.ac.cn
In our scheme, the single-photon transport can be controlled by a cavity field. When the control cavity field is in the vacuum state, our scheme can be considered as a 1D waveguide coupled to a two-level system. Therefore, the input single photon will be reflected in the resonance case. When the control cavity contains one photon, our scheme becomes a 1D waveguide coupled to a three-level system with cascade configuration. The full transmission of the input single photon can be obtained in the resonance case. It is necessary to note that the control of single-photon transport by another photon without classical field in 1D waveguide with linear dispersion relation has been studied in Ref. 24]. We also study the single-photon transport controlled by the parameters of the control cavity, such as the coupling strength to the emitter, photon number and resonant frequency. By the way, the quantum control of single-photon transport in 1D waveguide with linear dispersion relation by cavityemitter coupling strength has been studied in Ref. 25]. Our scheme does not contain inelastic scattering. Our scheme can be considered as a $1 \mathrm{D}$ waveguide with nonlinear dispersion relation coupled to a V-type atom in the dressed-state representation. Some outcomes can be understood better than in the bear-state representation.

\section{MODEL AND HAMILTONIAN}

The schematic diagram of the considered system is shown in Fig. 1. The 1D waveguide is composed by a coupled-cavity array with a very large number of singlemode cavities and one two-mode cavity. The cavity modes of the 1D coupled cavities are represented by the annihilation operators $a_{j}$, with $j$ the label of site. Here we take the site of the two-mode cavity 0 . Hence, one mode of the two-mode cavity, which is coupled to the nearest-neighbor single-mode cavities, is represented by the annihilation operators $a_{0}$. We represent the other mode of the two-mode cavity by the annihilation opera- 


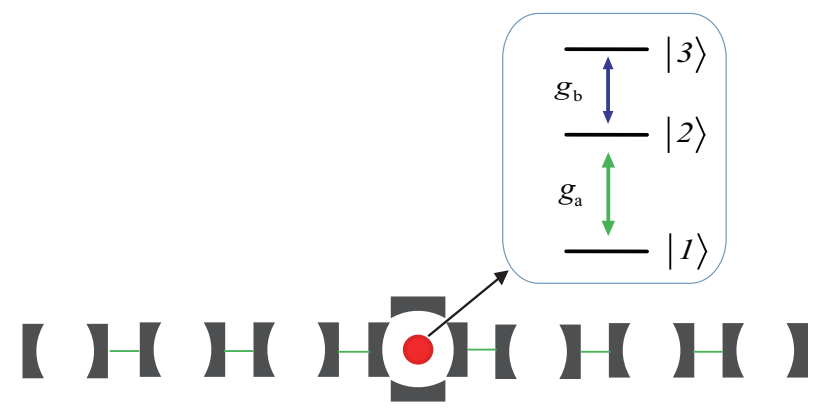

FIG. 1: Schematic diagram of the single-photon transport in a one-dimensional coupled-resonator waveguide. A three-level system with cascade configuration is coupled to the waveguide.

tor $b$. For simplicity, we assume that the cavity modes $a_{j}$ have the same resonant frequency $\omega_{a}$. The cavity mode $b$ has the resonant frequency $\omega_{b}$. A three-level system in cascade configuration is doped in the two-mode cavity. The three-level system can be a real atom or a manual atom-like object. It will be mentioned as an atom below. We represent atomic states $|1\rangle,|2\rangle$ and $|3\rangle$, with frequencies $\omega_{1}, \omega_{2}$ and $\omega_{3}$, respectively. We choose the groundstate energy for reference and ,hence, take $\omega_{1}$ zero. The level transitions between $|1\rangle \leftrightarrow|2\rangle$ and $|2\rangle \leftrightarrow|3\rangle$ are coupled to the modes $a_{0}$ and $b$ with strengths $g_{a}$ and $g_{b}$, respectively. In the rotating-wave approximation, the system Hamiltonian has the form of

$$
H=H_{C}+H_{A}+H_{I},
$$

with

$$
\begin{aligned}
H_{C} & =\omega_{a} a_{j}^{\dagger} a_{j}+\omega_{b} b^{\dagger} b-\xi \sum_{j}\left(a_{j+1}^{\dagger} a_{j}+\text { h.c. }\right), \\
H_{A} & =\omega_{2} \sigma^{22}+\omega_{3} \sigma^{33} \\
H_{I} & =g_{a} \sigma^{21} a_{0}+g_{b} \sigma^{32} b+h . c . .
\end{aligned}
$$

Here we have taken $\hbar=1$. The Hamiltonian $H_{C}$ denotes the cavity photons, $H_{A}$ is the atomic free Hamiltonian and $H_{I}$ describes the interaction of the atom with the two-mode cavity. The sum part of $H_{C}$ describes the hopping of the $a_{j}$ mode photons to the nearest-neighbor cavities with strength $\xi$. Here we have assumed that all the hopping strengths are equal. By introducing the Fourier transform $a_{k}=\frac{1}{\sqrt{N}} \sum_{k} e^{i k j}$ and taking the distances between two nearest-neighbor cavities unit, the term $\omega_{a} a_{j}^{\dagger} a_{j}-\xi \sum_{j}\left(a_{j+1}^{\dagger} a_{j}+\right.$ h.c. $)$ can be diagonalized as $\sum_{k} \Omega_{k} a_{k}^{\dagger} a_{k}$, with $\Omega_{k}=\omega_{a}-2 \xi \cos k$ and $N$ being the cavity number. This implies the nonlinear dispersion relation of the $1 \mathrm{D}$ coupled-cavity waveguide.

\section{CONTROL OF SINGLE-PHOTON TRANSPORT}

We assume that, initially, a photon is injected into the waveguide from the left side, the atom is in its ground state $|1\rangle$, and that the two-mode cavitiy contains $n b$ mode photons. We note that the value of $n$ can not be very large, i. e. $g_{b} \sqrt{n} \ll\left\{\omega_{2}, \omega_{3}\right\}$. This because we have emploied the rotating-wave approximation in Hamiltonian (1). The input single photon will transport along the waveguide and be scattered due to the atom-cavity interaction. Obviously, when $n=0$, the atom will absorb the injected photon, meanwhile making a transition from the level $|1\rangle$ to $|2\rangle$, and then reemit a photon, meanwhile making a transition from the level $|2\rangle$ to $|1\rangle$. In this case, the atomic level $|3\rangle$ never participates in the dynamic process because the atomic transition from level $|2\rangle$ to $|3\rangle$ needs to absorb a $b$-mode photon. Hence, our scheme is equal to a coupled-cavity waveguide coupled to a twolevel system when $n=0$. However, when $n \neq 0$, the transition $|2\rangle \leftrightarrow|3\rangle$ participates in the dynamic process, revealing differen behaviors from the $n=0$ case due to the quantum interference between different atomic transitions.

The arbitrary state governed by the Hamiltonian (1) can be written as

$$
|\Psi\rangle=\sum_{j} \alpha_{j} a_{j}^{\dagger}|1, n\rangle|\phi\rangle+\beta|2, n\rangle|\phi\rangle+\zeta|3, n-1\rangle|\phi\rangle,
$$

with $\alpha_{j}, \beta$ and $\zeta$ probability amplitudes. The state $|m, n\rangle$, denotes that the atom is in the state $|m\rangle$, and the two-mode cavity contains $n b$-mode photons. The state $|\phi\rangle$ denotes the 1D waveguide does not contain any $a_{j}$-mode photon. From the eigenequation $H|\Psi\rangle=E|\Psi\rangle$, we can obtain a set of equations of the probability amplitudes. Then by eliminating the probability amplitudes $\beta$ and $\zeta$, we can obtain the equation of the probability amplitude $\alpha_{j}$, which reveals the single photon transport property, as

$$
\left[E-\left(\omega_{a}+n \omega_{b}\right)-V \delta_{j, 0}\right] \alpha_{j}=-\xi\left(\alpha_{j+1}+\alpha_{j-1}\right),
$$

with

$$
V=\frac{g_{a}^{2}\left\{E-\left[\omega_{3}+(n-1) \omega_{b}\right]\right\}}{\left[E-\left(\omega_{2}+n \omega_{b}\right)\right]\left\{E-\left[\omega_{3}+(n-1) \omega_{b}\right]\right\}-g_{b}^{2} n} .
$$

The effective potential $V$ resulting from the atom-cavity interaction located at site $j=0$ modifies the singlephoton transport property. If the two-mode cavity does not contain $b$-mode photons, we find $V=\frac{g_{a}^{2}}{E-\omega_{2}}$, in line with the outcome in Ref. 2]. We consider the simple case that the atomic transition $|1\rangle \leftrightarrow|2\rangle$ is driven resonantly by the input photon. The potential is derived as $V \rightarrow \infty$ when $n=0$. However, if we inject a $b$-mode photon into the two-mode cavity initially, i.e. $n=1$, the potential is obtained as $V=-\frac{g_{a}^{2}\left(E-\omega_{3}\right)}{g_{b}^{2}}$. Especially, when the $b$ mode photon resonantly drives the atomic transition, i.e. $\omega_{32}=\omega_{b}$, the potential equals to zero. Therefore, we can modify the effective potential between $\infty$ and 0 by one $b$-mode photon. This implies that the single-photon transport can be controlled by only one $b$-mode photon. 
For the single-photon transport in the $1 \mathrm{D}$ waveguide, the spatial dependence of the amplitude $\alpha_{j}$ can be expressed as

$$
\alpha_{j}=\left(e^{i k j}+r e^{-i k j}\right) \theta(-j)+t e^{i k j} \theta(j),
$$

where $r$ is the reflection amplitude and $t$ is the transmission amplitude. The Heaviside step function $\theta(x)$ equals to 1 when $x$ is larger than 0 , while it equals to 0 when $x$ is smaller than 0 . From equation (3) and (4), when $|j|>1$, we can find $E=n \omega_{b}+\omega_{a}-2 \xi \cos k_{a}$, which corresponds to the dispersion relation derived above. And when $j=0$, we can derive the expression of the reflection and transmission amplitudes as

$$
\begin{aligned}
r & =\frac{-g_{a}^{2}\left(\delta_{a}+\delta_{b}\right)}{2 i \xi\left[\delta_{a}\left(\delta_{a}+\delta_{b}\right)-g_{b}^{2} n\right] \sin k+g_{a}^{2}\left(\delta_{a}+\delta_{b}\right)}, \\
t & =\frac{2 i \xi\left[\delta_{a}\left(\delta_{a}+\delta_{b}\right)-g_{b}^{2} n\right] \sin k}{2 i \xi\left[\delta_{a}\left(\delta_{a}+\delta_{b}\right)-g_{b}^{2} n\right] \sin k+g_{a}^{2}\left(\delta_{a}+\delta_{b}\right)},
\end{aligned}
$$

with the detunings $\delta_{a}=\omega_{2}-\Omega_{k}$, and $\delta_{b}=\omega_{32}-\omega_{b}$. Here we have employed the continuity condition $\alpha_{0}^{+}=\alpha_{0}^{-}$. The relation $|r|^{2}+|t|^{2}=1$ can be easily verified. Obviously, when $\delta_{a}+\delta_{b}=0$ and $n \neq 0$, the full transmission of the input single photon is obtained due to the interferences. When $\delta_{a}\left(\delta_{a}+\delta_{b}\right)=g_{b}^{2} n$, the input single photon is completely reflected.

To show the details of the single photon transport property, we plot the reflection and transmission coefficients as a function of the momentum $k$ in Fig. 2 for various values of $\delta_{b}$ and $n$. The lines show rich shapes by adjusting the $b$-mode photons. The region of $k$ from $-\pi$ to $\pi$ is enough due to the fact that $r$ and $t$ are periodic functions of $k$ with the same period $2 \pi$.

From the expression (5), the single-photon transport property relates to the number of the $b$-mode photons. We first study the resonance case. When the two-mode cavity does not contain $b$-mode photons, we can find $r=-\frac{g_{a}^{2}}{2 i \xi \sin k \delta_{a}+g_{a}^{2}}$ and $t=\frac{2 i \xi \sin k \delta_{a}}{2 i \xi \sin k \delta_{a}+g_{a}^{2}}$. Obviously, when $\delta_{a}=0$, the input single photon will be completely reflected. This outcome has been obtained in a waveguide coupled to a two-level system [2]. However, when the two-mode cavity contains one or more $b$-mode photons resonantly driving the atomic transition $|2\rangle \leftrightarrow|3\rangle$, i.e. $\delta_{a}=\delta_{b}=0$ and $n>0$, the full transmission is achieved. We note that in the $n>1$ case, the single-photon transport is not affected by the number of $b$-mode photons in the resonance case. Therefore, the single-photon switch can be achieved by only one $b$-mode photon.

In the off-resonance case, the single-photon transport relates to the number of $b$-mode photons even when $n>1$. Here we assume that $g_{a}$ and $g_{b}$ have the same order of magnitude. This assumption is reasonable in experiment. It can be seen that when the number of $b$-mode photons is large enough, the nearly full transmission of single photon can be obtained. This can be understood from that $g_{b} \sqrt{n}$ is the effective coupling strength of the transition $|2, n\rangle \leftrightarrow|3, n-1\rangle$. When $n$ is large enough,
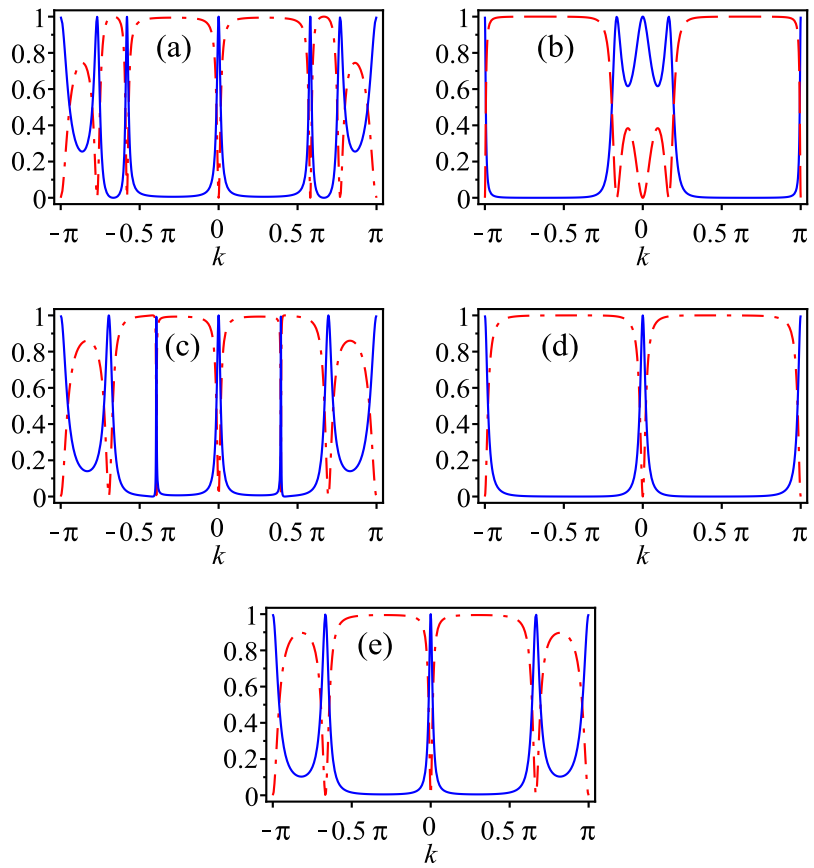

FIG. 2: The reflection and transmission coefficients against the momentum $k$ for various values of $\delta_{b}$ and $n$. The blue solid and red solid-dot lines represent the reflection and transmission coefficients, respectively. We take $n=1$ in (a) and (c), and $n=30$ in (b) and (d). In (a) and (b), $\delta_{b}=0$. In (c) and (d), $\delta_{b}=-3$. We take $n=0$ in (e). The other parameters are $g_{b}=1, \omega_{2}-\omega_{a}=2, \xi=2$. All the parameters but $n$ are in units of $g_{a}$.

the coupling strength to the transition $|2\rangle \leftrightarrow|3\rangle$ is much larger than the strength to $|1\rangle \leftrightarrow|2\rangle$. These behaviors can be understood better in the dressed representation as shown below.

Obviously, the single-photon transport property is affected by other parameters of the $b$-mode cavity, such as the resonant frequency and atom-cavity coupling strenghs. In Fig. 3a and 3b, we plot the reflection and transmission coefficients against the detuning $\delta_{b}$ when the two-mode cavity contains $b$-mode photons and the input photon is off-resonant to the transition $|1\rangle \leftrightarrow|2\rangle$. The detuning $\delta_{b}$ can be adjusted by the atomic transition frequency and the cavity resonant frequency. The control of single photon transport by tuning the resonant frequency of cavities has been studied in [18]. In our scheme, we bring in an extra cavity which is not in the array of the coupled cavities and is connected with the array by the three-level emitter. The line shapes are like Fano and anti-Fano shapes. This is in line with the conditions of full transmission and reflection obtained below Eqs. (5). Fig. $3 \mathrm{c}$ is the the reflection and transmission coefficients against the parameter $g_{b}^{2} n$. The feasibly controllable coupling strength between the cavity and the quantum dot has been proposed in Ref. [25]. 
(a)

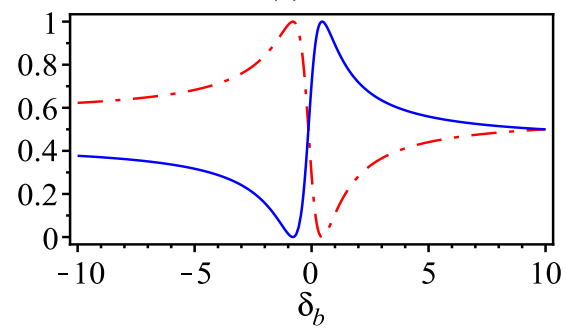

(b)

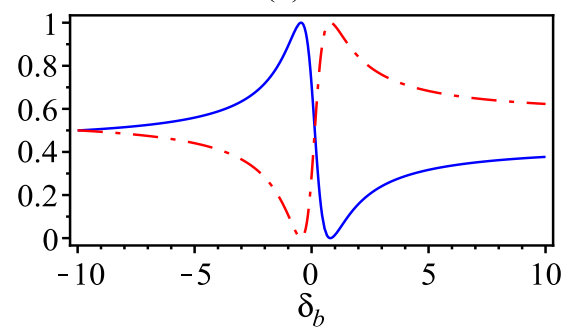

(c)

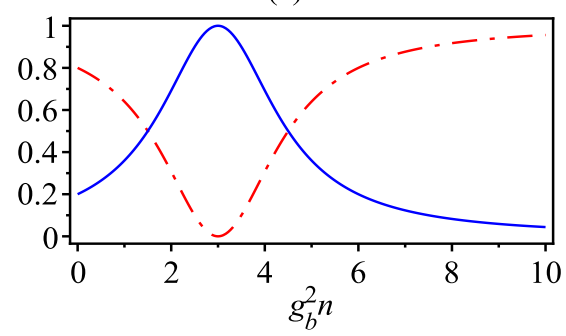

FIG. 3: The reflection and transmission coefficients against the detuning $\delta_{b}$ and $g_{b}^{2} n$. The blue solid and red soliddot lines represent the reflection and transmission coefficients,respectively. (a) and (b) are the coefficients against $\delta_{b}$. We take $\delta_{a}=0.8$ in (a) and $\delta_{a}=-0.8$ in (b). The other parameters of (a) and (b) are $g_{b}=1, k=\pi / 4, \xi=1, n=1$. (c) is the coefficients against $g_{b}^{2} n$. The parameters of (c) are $g_{b}=1, k=\pi / 6, \xi=2, \delta_{a}=1$ and $\delta_{b}=2$. All the parameters but $n$ are in units of $g_{a}$.

\section{DRESSED-STATE REPRESENTATION}

We can consider that the coupling between the $b$-mode photons and the atomic transition $|2\rangle \leftrightarrow|3\rangle$ leads to dressed states. The expression of the dressed states can be obtained as

$$
\left|\Psi_{ \pm}\right\rangle=A_{ \pm}|2, n\rangle+B_{ \pm}|3, n-1\rangle,
$$

with the corresponding energies

$$
\omega_{ \pm}=\omega_{2}+n \omega_{b}+\frac{\delta_{b} \pm \sqrt{\delta_{b}^{2}+4 g_{b}^{2} n}}{2} .
$$

Here $\quad A_{ \pm}=\frac{-\delta_{b} \pm \sqrt{\delta_{b}^{2}+4 g_{b}^{2} n}}{\sqrt{2 \sqrt{\delta_{b}^{2}+4 g_{b}^{2} n}\left(\sqrt{\delta_{b}^{2}+4 g_{b}^{2} n} \mp \delta_{b}\right)}}$ and $B_{ \pm}=$ $\frac{2 g_{b} \sqrt{n}}{\sqrt{2 \sqrt{\delta_{b}^{2}+4 g_{b}^{2} n}\left(\sqrt{\delta_{b}^{2}+4 g_{b}^{2} n} \mp \delta_{b}\right)}}$. Thus, our scheme can be

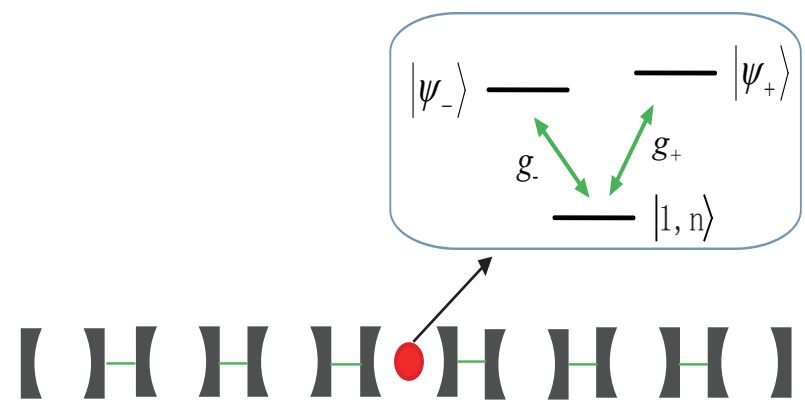

FIG. 4: In the dressed-state representation, the scheme shown in Fig. 1 can be considered as a $1 \mathrm{D}$ waveguide coupled to an effective V-type atom with effective coupling strengths $g_{ \pm}$.

considered as a waveguide coupled to a V-type system in the dressed-state representation, as shown in Fig. 4. The effective coupling strength of the $a_{0^{-}}$ mode photon to the transition $|1, n\rangle \leftrightarrow\left|\Psi_{ \pm}\right\rangle$can be derived as $g_{ \pm}=g_{a} A_{ \pm}$. Hence, the Hamiltonian represented in the dressed-state representation has the form of $H_{C}=\sum_{i= \pm} \omega_{i} \sigma^{i i}+\omega_{a} a_{j}^{\dagger} a_{j}-\xi \sum_{j}\left(a_{j+1}^{\dagger} a_{j}+\right.$ h.c. $)+$ $\sum_{i= \pm}\left(g_{i} a_{0}\left|\Psi_{i}\right\rangle\langle 1, n|+h . c.\right)$. Especially, When $n=0$, we can find $A_{ \pm}=1$ and $B \pm=0$, and the effective V-type system becomes a two-level system.

It is necessary to bring in two parameters, $\delta_{ \pm}=$ $\delta_{a}+\frac{\delta_{b} \pm \sqrt{\delta_{b}^{2}+4 g_{b}^{2} n}}{2}$, to represent the detunings between the transition energy $|1, n\rangle \leftrightarrow\left|\Psi_{ \pm}\right\rangle$and the energy $\Omega_{k}$ of the incident photon. When one of the two detunings $\delta_{ \pm}$ is zero, we can find the potential $V \rightarrow \infty$, and the amplitude $t=0$. Therefore, for the single-photon transport in a coupled-cavity waveguide coupled to a V-type system, once one of the atomic transitions resonantly matches the photon, the input photon will be completely reflected. We note that the condition $\delta_{ \pm}=0$ is equivalent to the condition $\delta_{a}\left(\delta_{a}+\delta_{b}\right)=g_{b}^{2} n$ derived in the bear-state representation.

When $\frac{\delta_{+}}{\delta_{-}}=\frac{g_{+}^{2}}{g_{-}^{2}}$, we can find $\delta_{a}+\delta_{b}=0$. As mentioned above, the full transmission can be obtained in this case. Therefore, the full transmission of the injected single photon can be achieved when $\frac{\delta_{+}}{\delta_{-}}=\frac{g_{+}^{2}}{g_{-}^{2}}$ in a coupled-cavity waveguide coupled to a V-type atom. All these outcomes can also be verified by obaining the single-photon transport property of a waveguide with nonlinear dispersion relation coupled to a V-type atom as we have done in the bear-state representation. These behaviors are similar to the single-photon transport in a linear waveguide coupled to a V-type atom [22].

The effective energies $\omega_{ \pm}$of the dressed state relate to the number of the $b$-mode photons. When the value of $n$ is large enough, the detunings $\delta_{ \pm}$become much larger than the effective coupling strengths $g_{ \pm}$. In this case, the single-photon transmission efficient is approximately unit because the V-type system is nearly decoupled to the input single photon. 


\section{CONCLUSIONS}

The transport of the injected single photon in a 1D waveguide coupled to a three-level emitter with cascade configuration has been investigated. The atomic transition from $|2\rangle$ to $|3\rangle$ is driven by $b$-mode photons. When the emitter is in the ground state initially, whether the $b$-mode cavity contains photons determines the effective configuration of the emitter. Consequently, the singlephoton transport property depends on whether the $b$ mode cavity contains a photon or not. The single-photon transport can also be controlled by the parameters of the b-mode cavity. We also study the dressed-state representation. All the outcomes are obtained in the strong coupling regime. Our scheme represents an all-optical device operated at single-photon level.

\section{ACKNOWLEDGEMENT}

This work is supported by "973" program (2010CB922904), grants from Chinese Academy of Sciences, NSFC (11175248).
[1] J. T. Shen and S. Fan, Opt. Lett. 30, 2001 (2005); Phys. Rev. Lett. 95, 213001 (2005).

[2] L. Zhou, Z. R. Gong, Y. X. Liu, C. P. Sun, and F. Nori, Phys. Rev. Lett. 101, 100501 (2008);

[3] J. T. Shen and S. Fan, Phys. Rev. Lett. 98, 153003 (2007); Phys. Rev. A 76, 062709 (2007).

[4] D. Roy, Phys. Rev. Lett. 106, 053601(2011); Scientific Reports 3. 2337 (2013); Phys. Rev. A 83. 043823 (2011); Phys. Rev. B 81, 155117 (2010);

[5] D. Roy, N. Bondyopadhaya, Phys. Rev. A 89, 043806 (2014);

[6] E. Rephaeli and S. Fan, Phys. Rev. Lett. 108, 143602(2012);

[7] E. Kocabas, E. Rephaeli and S. Fan, Phys. Rev. A 85, 023817 (2012); Phys. Rev. A 84, 063832 (2011);

[8] S. Fan, S. E. Kocabas and J.-T. Shen, Phys. Rev. A 82, 063821 (2010);

[9] J.-T. Shen and S. Fan, Phys. Rev. A 82, 021802(R) (2010); Phys. Rev. A 79, 023837 (2009); Phys. Rev. A 79, 023838 (2009).

[10] P. Longo, P. Schmitteckert and K. Busch, Phys. Rev. Lett. 104, 023602 (2010);

[11] C. Martens, P. Longo and K. Busch, New J. Phys. 15, 083019 (2013); J. F. M. Werra, P. Longo, and K. Busch, Phys. Rev. A 87,063821 (2013);

[12] T. Shi, S. Fan and C. P. Sun, Phys. Rev. A 84. 063803 (2011); T. Shi and S. Fan, Phys. Rev. A 84, 063803 (2011);

[13] H. Zheng, D. J. Gauthier and H. U. Baranger, Phys. Rev. Lett. 107, 223601(2011); Phys. Rev. Lett. 111, 090502 (2013).

[14] H. Zheng, D. J. Gauthier and H. U. Baranger, Phys. Rev. A 85, 043832 (2012); Phys. Rev. A 82, 063816 (2010).

[15] H. Zheng, and H. U. Baranger, Phys. Rev. Lett. 110, 113601 (2013).

[16] J. F. Huang, J. Q. Liao, and C. P. Sun, Phys. Rev. A 87, 023822 (2013); J. Q. Liao and C. K. Law, Phys. Rev. A 82, 053836 (2010).

[17] Z. R. Gong, H. Lan, L. Zhou and C. P. Sun, Phy. Rev.
A 78, 053806 (2008).

[18] J. Q. Liao, et. al. Phys. Rev. A 80, 014301 (2009); Phys. Rev. A 80, 042304 (2010).

[19] C.-H. Yan, L.-F. Wei, W.-Z. Jia and J.-T. Shen, Phys. Rev. A 84, 045801 (2011).

[20] M-T Cheng, X.-S. Ma, M.-T. Ding, Y.-Q. Luo, and G.-X. Zhao, Phys. Rev. A 85, 053840 (2012).

[21] Z. H. Wang, Y. Li, D. L. Zhou, C. P. Sun and P. Zhang, Phy. Rev. A 86, 023824 (2012); Z. H. Wang, L. Zhou, Y. Li and C. P. Sun, Phy. Rev. A 89, 053813 (2014);

[22] D. Witthaut and A. S. S $\phi$ rensen, New J. Phys. 12, 043052(2010).

[23] Q. Li, L. Zhou, and C. P. Sun, Phys. Rev. A 89, 063810 (2014); J. F. Huang, T. Shi, C. P. Sun and F. Nori, Phys. Rev. A 88, 013836 (2013).

[24] L. Neumeier, M. Leib, and M. J. Hartmann, Phys. Rev. Lett. 111, 063601 (2013).

[25] C.-H. Yan, W.-Z. Jia and L.-F. Wei, Phys. Rev. A 89, 033819 (2014).

[26] L. Zhou, L. P. Yang, Y. Li, and C. P. Sun, Phys. Rev. Lett. 111, 103604 (2013); J. Lu, L. Zhou, L. M. Kuang, and F. Nori, Phys. Rev. A 89, 013805 (2014).

[27] M. Bradford, K. C. Obi, and J. T. Shen, Phys. Rev. Lett. 108, 103902 (2012); M. Bradford and J. T. Shen, Phys. Rev. A 85, 043814 (2012).

[28] W.-B. Yan, Q.-B. Fan, and L. Zhou, Phys. Rev. A 85, 015803 (2012); W.-B. Yan, J.-F. Huang, and H. Fan, Scientific Reports 3, 3555 (2013); W.-B. Yan, and H. Fan, Scientific Reports 4, 4820 (2014).

[29] I. C. Hoi et al., Phys. Rev. Lett. 107, 073601 (2011).

[30] B. Dayan et al., Science 319, 1062 (2008).

[31] E. Vetsch et al., Phys. Rev. Lett. 104, 203603 (2010).

[32] A.V. Akimov et al., Nature (London) 450, 402 (2007).

[33] M. Bajcsy et al., Phys. Rev. Lett. 102, 203902 (2009).

[34] T. M. Babinec et al., Nat. Nanotechnol. 5, 195 (2010).

[35] J. Claudon et al., Nature Photon. 4, 174 (2010).

[36] J. Bleuse et al., Phys. Rev. Lett. 106, 103601 (2011).

[37] A. Laucht et al., Phys. Rev. X 2, 011014 (2012). 